\title{
Arteria lusoria: A rare cause of chronic dysphagia
}

\author{
Domoina Harivonjy Hasina Laingonirina ${ }^{1}$ ( ) | Andry Lalaina Rinà Rakotozafindrabe ${ }^{1}$ | \\ Chantelli Iamblaudiot Razafindrazoto ${ }^{1}$ @ | Lova Hasina Ny Ony Narindra Rajaonarison ${ }^{2}$ | \\ Nitah Harivony Randriamifidy ${ }^{1}$ | Tovo Harimanana Rabenjanahary ${ }^{1}$ | \\ Soloniaina Hélio Razafimahefa $^{3}$ | Rado Manitrala Ramanampamonjy ${ }^{1}$
}

\author{
${ }^{1}$ Department of Gastroenterology, \\ University Hospital Joseph Raseta \\ Befelatanana, Antananarivo, \\ Madagascar \\ ${ }^{2}$ Department of Radiology, University \\ Hospital Joseph Ravoahangy \\ Andrianavalona, Antananarivo, \\ Madagascar \\ ${ }^{3}$ Department of Hepato- \\ Gastroenterology, University Hospital \\ Andrainjato, Fianarantsoa, Madagascar

\section{Correspondence} \\ Domoina Harivonjy Hasina \\ Laingonirina, Department of \\ Gastroenterology, University \\ Hospital Joseph Raseta Befelatanana, \\ Antananarivo, Madagascar. \\ Email: toavinjanahary@gmail.com
}

\begin{abstract}
Arteria lusoria is a rare cause of dysphagia in which dysphagia due to esophageal compression. The upper GI endoscopy does not bring significant element that can orient the diagnosis. The injected thoracic CT scan remains the key examination for the diagnosis of dysphagia lusoria and to characterize the defective artery.
\end{abstract}

\section{K E Y W O R D S}

aberrant right subclavian artery, dysphagia lusoria

\section{1 | INTRODUCTION}

Arteria lusoria is a rare vascular malformation. It remains a rare cause of dysphagia and should be considered in the presence of any dysphagia unexplained by the usual endoscopic and radiological examinations. Dysphagia lusoria is a swallowing disorder due to extrinsic compression of the esophagus by the aberrant right subclavian artery or arteria lusoria. ${ }^{1}$ It is the most frequent vascular malformation with an incidence of $0.4 \%-2 \%$. $^{2}$ Its relationship with dysphagia is rarely described. Our objective was to report the case of a 70-year-old female patient with chronic dysphagia secondary to an aberrant right subclavian artery or dysphagia lusoria.

\section{2 | CASE REPORT}

A 70-year-old woman presented to a consultation for dysphagia evolving for more than 10 years, mainly on solid foods. There was no obvious blockage on swallowing. The general condition was still preserved. Moreover, the dysphagia was associated with spontaneous chest pain, which was favored by swallowing. There was no vomiting or transit disorder. The patient had a history of intestinal amoebiasis treated in 2010 and chronic smoking weaned in 2014 (10 pack-years). There was no personal history of alcoholism or family history of systemic disease.

On clinical examination, the patient was conscious and apyretic, and blood pressure was 130/80 $\mathrm{mmHg}$. She had 
a performance status score at 0 and a body mass index $18 \mathrm{~kg} / \mathrm{m}^{2}$. The respiratory examination was normal. The cardiovascular examination did not show any particular abnormally, especially vascular murmurs. There were no palpable peripheral nodes.

Biological examinations such as complete blood count, erythrocyte sedimentation rate, C-reactive protein, fasting glycemia, creatinine, urea, serum protein electrophoresis, serum calcium, TSH, free T3, and T4 were normal. Human immunodeficiency virus serology was negative.

Upper gastrointestinal (GI) endoscopy did not reveal any abnormality except for a slightly angled esophagus $25 \mathrm{~cm}$ from the dental arch. Step biopsy for eosinophilic esophagitis was normal. Chest X-ray came back normal. Chest and abdominal CT scan revealed a vascular image in favor of an aberrant right subclavian artery (Figures 1 and 2) and a left incidentaloma.

\section{DISCUSSION}

We report the first case of a dysphagia of rare cause, related to a vascular malformation. The aberrant right subclavian artery or arteria lusoria is a rare cause of dysphagia, which should be considered in the presence of any dysphagia unexplained by the usual endoscopic and radiological examinations. ${ }^{3-5}$ It is the most frequent malformation of the aortic arch and is sometimes associated with other malformations such as the presence of a bicarotid trunk or a tetralogy of Fallot. ${ }^{3,4}$ Arteria lusoria can occur not only in young subjects but also in older patients. We reported the case of a 70-year-old female patient who presented with dysphagia lusoria.
Dandelooy et al reported a similar case in a 76-year-old patient. ${ }^{6}$ However, Khnaba et al reported a younger patient aged 45 years. ${ }^{3}$

Arteria lusoria is asymptomatic in $90 \%$ of cases and discovered incidentally on radiological examinations performed in other contexts. ${ }^{2}$ Clinical signs are dominated by dyspnea due to tracheal compression and dysphagia due to esophageal compression as in our patient's case. Zapata et al described dysphagia with solids during arteria lusoria as in our patient's case. ${ }^{4}$

The upper gastrointestinal (GI) endoscopy showed in our patient a slightly bent esophagus, and there were no mucosal and parietal abnormalities. The upper GI endoscopy is the key examination to perform in case of dysphagia. It allows to search for organic causes of dysphagia and to eliminate tumor causes especially in older patients like our case. ${ }^{5}$ During lusoria dysphagia, upper GI endoscopy does not bring significant element that can orient the diagnosis but can sometimes reveal indirect signs of extrinsic compression. ${ }^{6}$

Radiological examinations play an important role in the etiological approach to dysphagia after upper GI endoscopy. The barium transit of the esophagus is indicated to look for esophageal stenosis. ${ }^{7}$ This examination was not performed in our case. Esophageal manometry may be performed depending on the clinical context and if the result of the upper GI endoscopy is normal. The injected thoracic CT scan remains the key examination for the diagnosis of dysphagia lusoria. It allows to search for extrinsic compression and to characterize the defective artery. Our patient had benefited from an injected thoracic scan showing esophageal compression of vascular origin by an aberrant right subclavian artery. According to Yang et al,

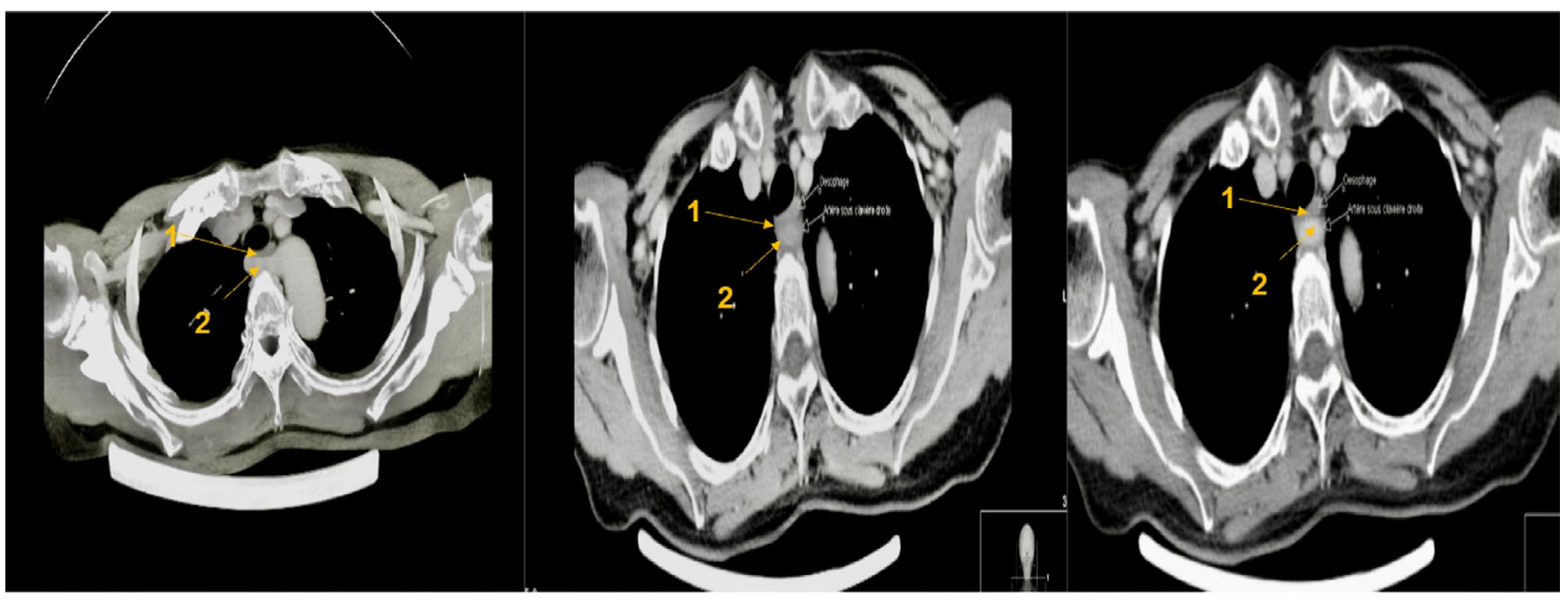

F I G U RE 1 Coronal section of thoracic CT scan of a 70-year-old woman shows a vascular image in favor of an aberrant right subclavian artery compressing the esophagus. 1: esophagus and 2: aberrant right subclavian artery 
FIGURE 2 Axial section of a thoracic CT scan of a 70-year-old woman shows a vascular image in favor of an aberrant right subclavian artery compressing the esophagus. 1: esophagus and 2: aberrant right subclavian artery
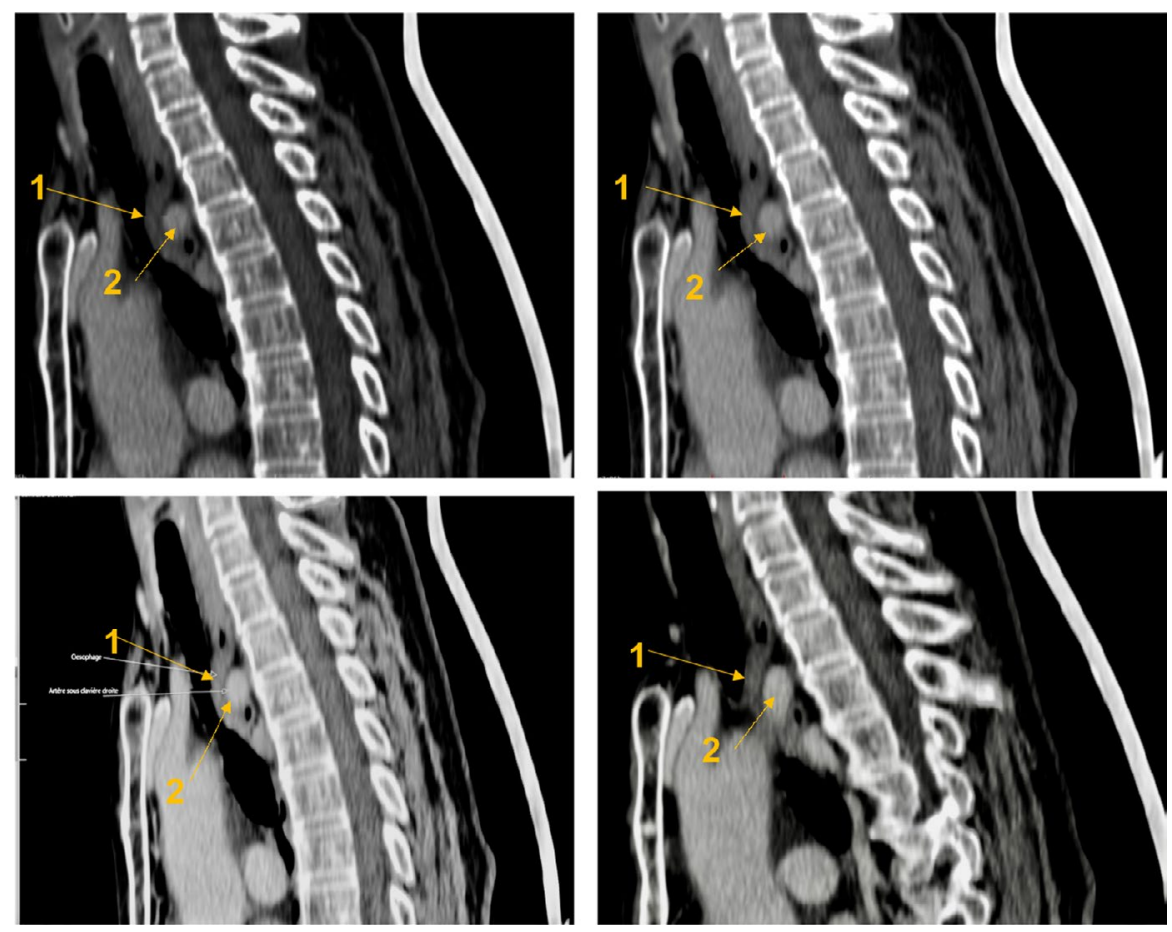

the sensitivity of the thoracic $\mathrm{CT}$ for the diagnosis of arteria lusoria is $100 \% .^{8}$ Arteria lusoria was confirmed by an injected chest CT scan showing compression of the esophagus by the aberrant right subclavian artery in the absence of obvious etiologies of dysphagia. Chest CT scan could also incidentally reveal the presence of an asymptomatic arteria lusoria. $^{8}$

In our case, a medical treatment was instituted associating the prescription of proton pump inhibitors and a modification of the food habits; the taking of semi-liquid meals was privileged. In the case of moderate symptoms without any impact on the patient's nutritional status, medical treatment with proton pump inhibitors combined with hygienic and dietary measures is indicated. ${ }^{9}$ The association with prokinetics has not shown any additional efficacy. Surgical treatment is discussed in the presence of severe symptoms with weight loss and undernutrition. It consists of reconstruction of the aberrant artery. The benefit-risk ratio must be evaluated before this procedure. The patient should be referred to an expert vascular surgery center. ${ }^{2-9}$

\section{CONCLUSION}

This is the first described case of arteria lusoria in Madagascar. This observation provides information on the existence of a rare cause of dysphagia of vascular origin. It should be considered before any dysphagia unexplained by the usual endoscopic and radiological examinations. It remains a diagnosis of elimination, which requires a thorough investigation before retaining the diagnosis. The treatment is essentially medical except in severe forms.

\section{ACKNOWLEDGEMENTS}

We gratefully acknowledge Andry Lalaina Rinà Rakotozafindrabe for providing this case report and Lova Hasina Ny Ony Narindra Rajaonarison for interpreting and providing the radiological image. The patient has given the consent for this publication.

\section{CONFLICT OF INTEREST}

None declared.

\section{AUTHOR CONTRIBUTIONS}

DHHL, ALRR, CIR, and LHNONR were the main contributors to the drafting of the manuscript. NHR, THR, SHR, and RMR involved in review and approval of final manuscript.

\section{ETHICAL APPROVAL}

The project was approved by the hierarchical heads of University Hospital Joseph Raseta Befelatanana, Antananarivo. Written consent was obtained from the patient for publication of this case report and accompanying images.

\section{CONSENT}

The patient has agreed and signed to give consent that his information and images will be published.

\section{DATA AVAILABILITY STATEMENT}

Data available on request from the corresponding author. 


\section{ORCID}

Domoina Harivonjy Hasina Laingonirina (1) https://orcid. org/0000-0002-4511-4584

Chantelli Iamblaudiot Razafindrazoto (1) https://orcid. org/0000-0002-5751-0373

\section{REFERENCES}

1. Bayford D. An account of a singular case of obstructed deglutition. Mem Med Soc Lond. 1794;2:275-286.

2. Di Serafino M, Severino R, Lisanti F, Rocca R, Scarano E. Dysphagia lusoria: an uncommon cause of dysphagia. $J$ Hepato Gastro Dis. 2016;2:134. https://doi.org/10.4172/2475-3181. 1000134

3. Khnaba S, Menany M, Moukinebillah M, Amil T, Radouane B. Arteria lusoria associee a un tronc bi-carotidien: à propos d'un cas et revue de la littérature. Pan Afr Med J. 2015;22:376.

4. Zapata H, Edwards JE, Titus JL. Aberrant right subclavian artery with left aortic arch: associated cardiac anomalies. Pediatr Cardiol. 1993;14(3):159-161.

5. Kantarceken B, Bulbuloglu E, Yuksel M, Cetinkaya A. Dysphagia lusorium in elderly: a case report. World J Gastro. 2004;10(16):2459-2460.
6. Dandelooy J, Coveliers JPM, Van Schil PEY, Anguille S. Dysphagia lusoria. CMAJ. 2009;181(8):498.

7. Lee KG, Lath N. Dysphagia lusoria-A rare cause of prolonged dysphagia. Med J Malaysia. 2015;70(1):52-53.

8. Yang M, Mo X-M, Jin J-Y, et al. Diagnostic value of 64 multislice CT in typing of congenital aortic anomaly in neonates and infants. Zhong Hua Yi Xue Za Zhi. 2010;90(31):2167-2171.

9. Munoz A, Obregon J, Elias J, Manuel J. Dysphagia lusoria: a case report and review of the literature. Rev Col Gastroenterol. 2009;24(4):396-402.

How to cite this article: Hasina Laingonirina DH, Rakotozafindrabe ALR, Razafindrazoto CI, et al. Arteria lusoria: A rare cause of chronic dysphagia. Clin Case Rep. 2021;9:e04895. https://doi. org $/ 10.1002 / \mathrm{ccr} 3.4895$ 